Population Study

Poster

Abstract ID: 86

\title{
Prevalence and intervention of malnutrition risk of Malaysian elderly living in the community: A literature review
}

Norain Zainudin | Aliza Haslinda Hamirudin | Nor Azlina A. Rahman | Suriati Sidek

International Islamic University Malaysia

Introduction: Malnutrition among the elderly is a concern around the globe, including in Malaysia due to its negative effects to health. The aims of this review are to identify prevalence of malnutrition risk in Malaysian elderly living in the community; and to identify the types of nutrition intervention provided to the elderly following malnutrition risk identification. Methods: Literature searches were undertaken using online databases and a manual search. Main keywords used for literature search strategy were malnutrition, elderly and Malaysia. Inclusion criteria for the literature searches are studies performed in Malaysian community, using either nutrition screening or assessment tool and elderly aged $\geq 60$ years old. Exclusion criteria are studied among hospitalized elderly, conducted in nursing homes, validation and development studies of nutrition screening tool. Results: Four published studies were eligible to be included in the review. The studies showed that prevalence of malnutrition in the Malaysian elderly living in the community is approximately $1.3 \%$ - $36.3 \%$. A range of $25.3 \%$ to $48.5 \%$ elderly was reported to be at malnutrition risk. All of the identified studies were cross-sectional studies; whilst none of the studies provided nutrition intervention to the elderly. Conclusions: Alarming rates of malnutrition risk of Malaysian elderly living in the community has been identified. Appropriate nutrition intervention for malnourished and at risk elderly should be targeted to improve nutritional status and prevent health deterioration. Further evaluation of effectiveness of nutrition intervention following malnutrition risk identification is warranted in future studies.

KEYWORDS: malnutrition, elderly, nutrition intervention, community 